ARTICLE INFO

Received

November 04, 2020

Revised

January 11, 2021

Accepted

January 13, 2021

Published

February 16, 2021

*Corresponding authors

Zichun Hua

E-mail

huazc@nju.edu.cn

Yang Wang

E-mail

wangyang@njust.edu.cn

Keywords

Autoinduction

Pseudomonas syringae

Lipodepsipeptide

Syringomycin

$v g b$ gene

How to cite

Li GY, Zhou TR, Wang YQ,

Wang Y, Hua ZC.

Autoinduction of Vitreoscilla

hemoglobin enhance the

production of syringomycin

from Pseudomonas syringae

HS191. Sci Lett 2021;

9(1):12-18
Open Access

\section{Autoinduction of Vitreoscilla Hemoglobin Enhanced Syringomycin from Production of syringae HS191}

\author{
Guiyao $\mathrm{Li}^{1}$, Taoran Zhou ${ }^{1}$, Yuqi Wang ${ }^{2}$, Yang Wang ${ }^{2 *}$, Zichun Hua ${ }^{1 *}$ \\ ${ }^{1}$ School of Life Science, Nanjing University, 210023, Nanjing, Jiangsu Province, China \\ 2 School of Environmental and Biological Engineering, Nanjing University of Science \\ and Technology, 210094, Nanjing, Jiangsu Province, China
}

\section{Abstract}

Syringomycin is a cyclic lipodepsipeptide produced by strains of Pseudomonas syringae. The potent herbicidal and fungicidal activities of syringomycin make it a promising compound for fungiostasis and weed control. However, the production of syringomycin from the wild-type strains is low. The discoveries that Pseudomonas syringae is aerobic, and the syringomycin synthetase $\mathrm{SyrB2}$ is an $\mathrm{O}_{2}$-dependent halogenase, led us to establish an autoinducible Vitreoscilla hemoglobin expression system for oxygen supply during fermentation, thereby increasing the yield of syringomycin. By employing the quorum sensing system for the expression of Vitreoscilla hemoglobin gene ( $\mathrm{vg} b)$, we found that Pseudomonas syringae HS191 that expressed $v g b$, facilitated cell growth and general biomass. Furthermore, syringomycin bioassay showed that the fungal inhibition zones increased from $2.5 \mathrm{~mm}$ to $3.2 \mathrm{~mm}$, and HPLC analysis confirmed that the expression of $v g b$ resulted in a $71.1 \%$ increase in syringomycin production compared to the wild-type strain. The Vitreoscilla hemoglobin has been widely applied to fermentation optimization; however, in the case of Pseudomonas, increased oxygen supply is only beneficial during the stationary phase, while a high concentration of oxygen inhibited the cell propagation during the logarithmic phase. Here we report the autoinduction of Vitreoscilla hemoglobin by engineering the quorumsensing system. This synthetic circuit significantly improved the syringomycin production. The Vitreoscilla hemoglobin-autoinduction system not only caters to the dynamic oxygen demand but also avoids inducer supplementation. 


\section{Introduction}

Vitreoscilla, an anaerobic gram-negative genus in the Vitreoscillaceae family, grows vigorously on rotting plants and other oxygen-intensive environments [1]. It is reported that Vitreoscilla can synthesize a protein Vitreoscilla hemoglobin $(\mathrm{VHb})$ under hypoxic conditions. $V H b$ works as an oxygenbinding protein that promotes oxygen delivery and reduces oxygen consumption when the dissolved oxygen is limited [2-4]. Therefore, $V H b$ will improve bacteria biomass and secretion products during the fermentation even at low oxygen concentration. The Vitreoscilla hemoglobin gene $(v g b)$ has been expressed in many kinds of bacteria and facilitated the growth of the host strains as well as the production of secondary metabolites [5-7]. Pseudomonas syringae is a rich source of secondary metabolites, including amphisin, syringomycin, syringopeptin, and so on [8, 9]. These cyclic lipopeptides are potent inhibitors of drug-resistant bacteria with low side effects [10]. Syringomycin is not only antagonistic against gram-positive pathogens like Bacillus anthracis, Staphylococcus aureus, Mycobacterium tuberculosis, etc. but also serves as a promising next-generation herbicide [11]. However, the production of syringomycin from $P$. syringae is too low to cater to industrial application.

Syringomycin fermentation is tightly related to fermentation medium, temperature, $\mathrm{pH}$ and dissolved oxygen [12]. Among those, oxygen supply plays a pivotal role in the production of syringomycin. Apart from the beneficial effects of oxygen on cell growth, the enzymes involved in the biosynthesis of syringomycin, syrB2, is an oxygendependent halogenase [13]. The reduction of oxygen concentration below $2 \%$ increases the lag period, while at $0.5 \%$ oxygen or lower, cell generation time during the logarithmic phase increases and cell yield at the stationary phase is reduced [14]. Hence, in time supply of oxygen during the stationary phase may facilitate the general cell yield. For this purpose, here we applied the quorum sensing mechanism for the autoinducible expression of $v g b$ gene in $P$. syringae HS191 to examine the effects of $V H b$ on biomass yield and syringomycin production.

\section{Materials and methods}

\section{Bacterial strains, plasmids and primers}

The bacterial strains Pseudomonas syringae pv. syringae HS191 was a gift from Dennis C. Gross (Texas A \& M University, Texas, United States of
America), Escherichia coli DH10B was purchased from ThermoFisher Scientific. The plasmids used in this study were: pLG-sfGFP, pMD18-T- $v g b$ and pGE-PBG. The services of primer synthesis and DNA sequencing were provided by Genwiz (Suzhou, China). The primers used are presented in Table 1.

\section{Culture and growth conditions}

The strain $P$. syringae HS191 was cultured at $28^{\circ} \mathrm{C}$ in nutrient broth yeast extract (NBY) medium [15], and $E$. coli $\mathrm{DH} 10 \mathrm{~B}$ strain was cultured at $37{ }^{\circ} \mathrm{C}$ in Luria-Bertani (LB). When required, antibiotics were added to the growth media at the following concentrations: ampicillin, $100 \mu \mathrm{g} / \mathrm{ml}$; kanamycin, $50 \mu \mathrm{g} / \mathrm{ml}$.

\section{DNA manipulation techniques}

DNA manipulation and E. coli transformation were performed using standard techniques [16]. $P$. syringae transformation was performed as described previously [17]. Restriction enzymes, T4ligases, Q5® high-fidelity DNA polymerase, Gibson Assembly ${ }^{\circledR}$ master mix, and DNA markers were purchased from New England Biolabs (NEB).

\section{Construction of the sfGFP reporter strain}

The polymerase chain reaction (PCR) amplified the genomic fragment harboring syrP-syrB1 using primer pair: syrPBFor/syrPBRev and TA cloned into pGEMT easy, forming pGE-syrPB. In the next step, PCR amplified the vector pGE-syrPB using primers pGE-syrPBFor/pGE-syrPBRev. In another step, PCR amplified sfGFP from pLG-sfGFP using primers sfGFPFor /sfGFPRev and then PCR amplified genR from pTA-mob using primer pair genRFor/genRRev. Gibson assembled the above three fragments to form plasmid pGE-PBG. The plasmid pGE-PBG was digested with EcoRI to purify the $4617 \mathrm{bp}$ recombinant fragment syrPsfGFP-genR-syrB1, which was then transformed into strain HS191. The colonies that survive on gentamycin and demonstrated green fluorescence were verified by PCR analysis using primer pair genRfor/syrB2Rev, and the correct mutant was named HS191-sfGFP.

\section{Construction of pLG-vgb}

The $v g b$ gene was cloned from the pMD18-T- $v g b$ plasmid by PCR using primer pair vgbFor and vgbRev. Meanwhile, the expression vector was amplified from pLG-sfGFP using primer pair then LGFor and LGRev. The above two fragments were 
Table 1 List of primers used in this study.

\begin{tabular}{|c|c|}
\hline Primers & Sequence $\left(5^{\prime} \sim 3{ }^{\prime}\right)$ \\
\hline syrPBFor & $5^{\prime}$-ggcggtttcactggatatcg-3' \\
\hline syrPBRev & 5 '-cgtgatgagtcaccggcagttc-3' \\
\hline pGE-syrPBFor & 5 '-gtggattaatagactggatgccgattaagaacactgac-3' \\
\hline pGE-syrPBRev & 5 '-gttcttctcctttgctcatgaccaaggctcctgtgt-3, \\
\hline sfGFPFor & 5'-acacaggagccttggtcatgagcaaaggagaagaac-3' \\
\hline sfGFPRev & $5^{\prime}$-cgtttccacggtgtgcgtctagatcagctaattaagc-3' \\
\hline genRFor & 5 '-gcttaattagctgatctagacgcacaccgtggaaacg-3, \\
\hline genRRev & 5 '-gtcagtgttcttaatcggcatccagtctattaatccac-3, \\
\hline syrB2RFor & 5 '-caacgcgcttggtgcttatgtg-3' \\
\hline syrB2Rev & $5^{\prime}$-ctcggacctaatgtgctctg-3', \\
\hline$v g b$ For & 5'-cattaaagaggagaaaggtaccAtgttagaccagcaaaccattaac-3' \\
\hline$v g b \operatorname{Rev}$ & 5 '-gatcagctaattaagcttttattcaaccgcttgagcgtac-3' \\
\hline LGFor & 5 '-gtacgctcaagcggttgaataaaagcttaattagctgatc-3' \\
\hline LGRev & $5^{\prime}$-gttaatggtttgctggtctaacaTggtacctttctcctctttaatg-3' \\
\hline
\end{tabular}

ligated by Gibson assembly, and the plasmid formed was designated pLG- $v g b$. Finally, the plasmid pLG-vgb was transformed into strain HS191, and the mutant was named HS191-vgb.

\section{Bioassay for syringomycin}

The production of syringomycin by $P$. syringae HS191 and derivative mutants were evaluated using a bioassay previously described for syringomycin production on Hrp minimal medium (HMM) agar [18] with minor modifications. Bacterial strains were grown overnight in $3 \mathrm{ml}$ $\mathrm{NBY}$ at $28^{\circ} \mathrm{C}$ with shaking at $180 \mathrm{rpm}$. Cells were washed and resuspended in sterile deionized water to $\mathrm{OD}_{600}=0.3\left(\sim 2 \times 10^{8} \mathrm{CFU} / \mathrm{ml}\right)$, and $5 \mu 1$ aliquots of bacterial suspension were spotted on HMM agar medium. After an incubation period of 3 days at $28^{\circ} \mathrm{C}$, the plates were lightly sprayed with a cell suspension of Geotrichum candidum strain AS2.616 using a sterile chromatography sprayer. After $24 \mathrm{~h}$, quantification of syringomycin production was determined by measuring the diameter of inhibition zones and compared with the wild type strain of HS191. This experiment was conducted in triplicate.

\section{Analysis of biomass and syringomycin yield}

Single colonies of HS191-vg $b$ and wild-type were inoculated into $\mathrm{LB}$ medium and cultured at $28^{\circ} \mathrm{C}$ with shaking at $200 \mathrm{rpm}$ till logarithmic phase (24 h). Then $5 \mathrm{ml}$ of the logarithmic phase bacteria were inoculated in $100 \mathrm{ml}$ fresh LB in 250-ml flask under the same conditions for 5 days till the stationary growth phase. The cells after fermentation were harvested at $4000 \mathrm{rpm}$ for $10 \mathrm{~min}$ at $4^{\circ} \mathrm{C}$, then the biomass of the fermentation culture was calculated according to the dry weight. After $48 \mathrm{~h}$ incubation of the HS191-vgb and wild-type, the cultures were mixed with an equal volume of acidified acetone, and the mixtures were centrifuged at $5000 \mathrm{rpm}$ for 10 min [19]. The supernatants were collected and concentrated to $1 / 10$ volume at $45^{\circ} \mathrm{C}$ to $50^{\circ} \mathrm{C}$ with a rotary evaporator. The dried samples were dissolved in water: acetone (40:60 v/v) and subjected to high-performance reverse-phase liquid chromatography on a $1 \times 25 \mathrm{~cm}, \mathrm{C} 18$ column (Alltech Associates, Inc.) with a 2-propanol gradient $(0-100 \%$ in $35 \mathrm{~min}, 0.1 \%$ TFA $-0.1 \%$ TFA in 2-propanol). The syringomycin peak was identified by bioassay and collected.

\section{Results}

\section{Construction of HS191-sfGFP and HS191-GV}

To establish a real-time gene expression platform for the monitoring of the syringomycin synthesis, we integrated the sfGFP-ssr reporter gene in-frame into the immediate downstream of PsyrB1 promoter, which is the main promoter that drives the synthesis of syringomycin. The mutants that survived on NBY medium with gentamycin were not green until $20 \mathrm{~h}$ after incubation at $28^{\circ} \mathrm{C}$, and the green fluorescence faded after another $10 \mathrm{~h}$. The positive colonies were randomly selected for sequencing, and the correct mutant was designated as HS191sfGFP. Meanwhile, the autoinducible $v g b$ expressing plasmid was constructed by replacing the $s f G F P$ gene of pLG-sfGFP gene with $v g b$ that was derived from pMD18-T-vgb, using Gibson assembly. The transformants were miniprepped for verification with SpeI digestion, and the correct plasmid was named pLG-vgb. Finally, the plasmid pLG- $v g b$ was then transformed into cells of HS 191sfGFP to form strain HS191-GV. 

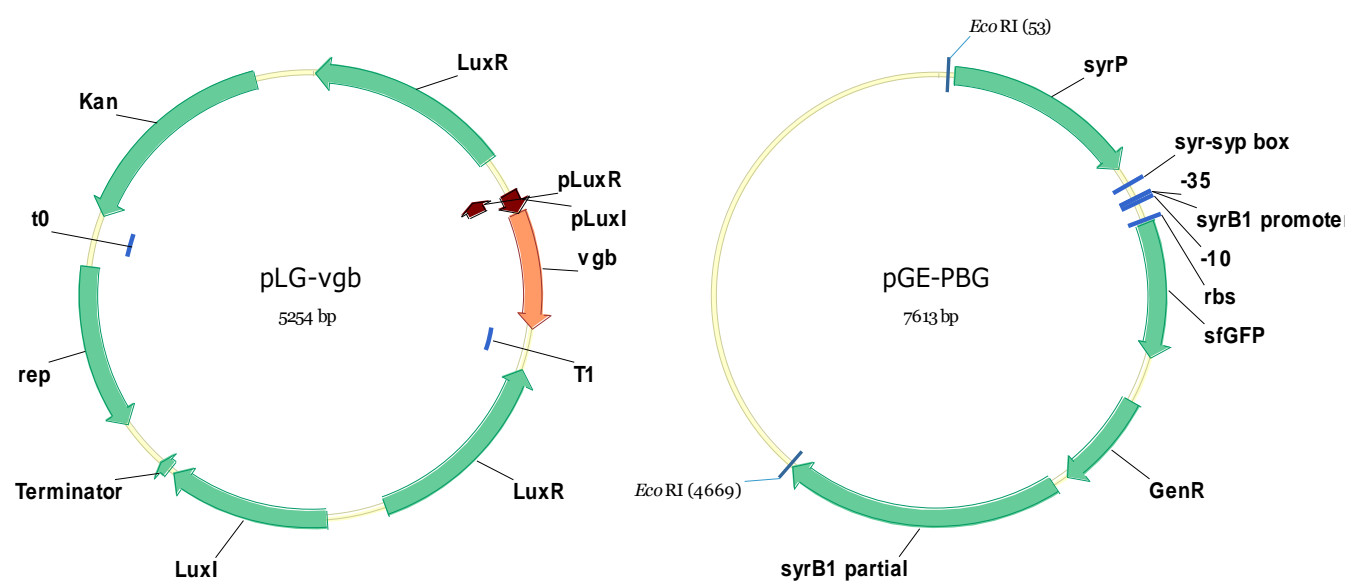

Fig. 1 Schematic map of plasmid pLG- $v g b$ and pGE-PBG. The exogenous genes including $v g b$ gene, kanamycin gene and promoter system Plux were integrated into HS191 by the plasmid pLG- $v g b$. The reporter gene sfGFP and antibiotic gene GenR were inserted into HS191 genome by plasmid pGE-PBG.

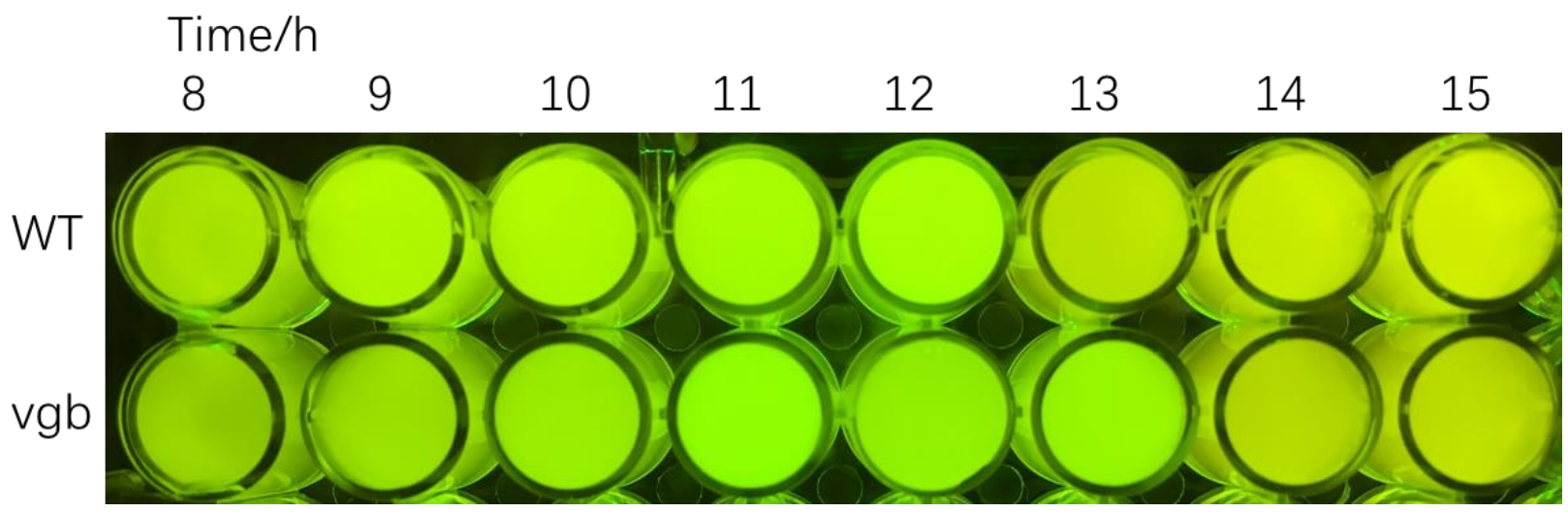

Fig. 2 GFP changes in the cultures of HS191-vgb and wild-type. The wild-type strain lost GFP after $12 \mathrm{~h}$ due to the degradation of GFP during the stationary phase. HS191-vgb lost GFP after $13 \mathrm{~h}$, because its logarithmic growth phase is a little longer. The results suggested that the Plux system works well in Pseudomonas syringae.
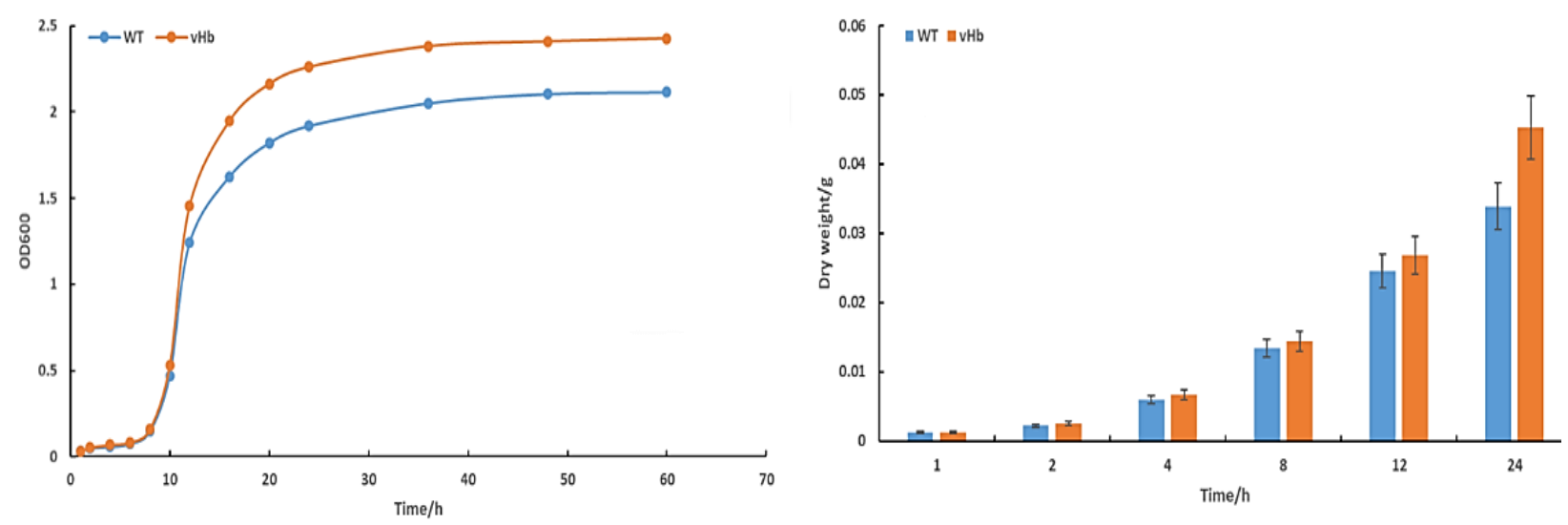

Fig. 3 The growth and biomass of HS191-vg $b$ and wild-type strains. The wild-type strain was cultured in LB medium at $28{ }^{\circ} \mathrm{C}$, $220 \mathrm{rpm}$, and the HS191-vgb was cultured in LB medium with kanamycin at $28^{\circ} \mathrm{C}, 220 \mathrm{rpm}$. For the growth records, one ml culture samples were used to detect the absorbance at $600 \mathrm{~nm}$. For the biomass, five $\mathrm{ml}$ culture samples were used to weigh after lyophilization. 
Expression of $\mathrm{VHb}$ extended the expression of syringomycin gene cluster

As the ssr tag facilitates the degradation of sfGFP, the PsyrB1-sfGFP-ssr serves as an ideal reporter system for the real-time detection of syringomycin synthesis. The expression profile of syringomycin gene cluster was monitored according to the green fluorescence of strain HS191-sfGFP and HS191GV. As shown in Fig. 2, There was no significant difference between the start point of the above two strains that appeared green fluorescence. However, strain HS191-GV maintained fluorescence for about $6 \mathrm{~h}$, while the $v g b$-negative strain HS191sfGFP lasted for only $5 \mathrm{~h}$.

\section{Expression of $\mathrm{VHb}$ increased the growth and biomass of strain HS191}

To evaluate the effects of $V H b$ on the growth of HS191, the growth curves of HS191-vgb and the wild-type strain were plotted, and the dry weight biomass was periodically recorded. As shown in Fig.

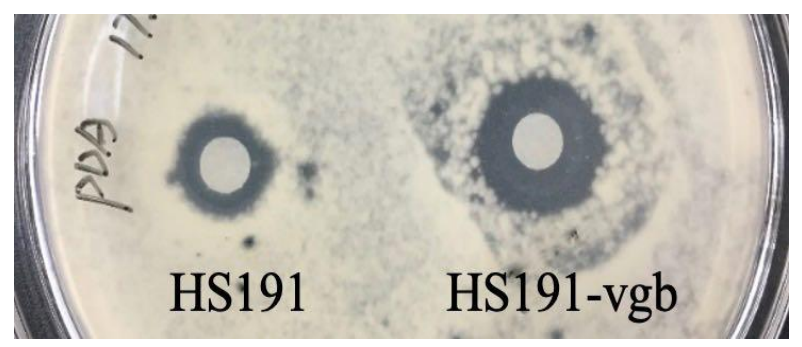

Fig. 4 Bioassay of HS191-vgb and wild-type to Geotrichum candidum.

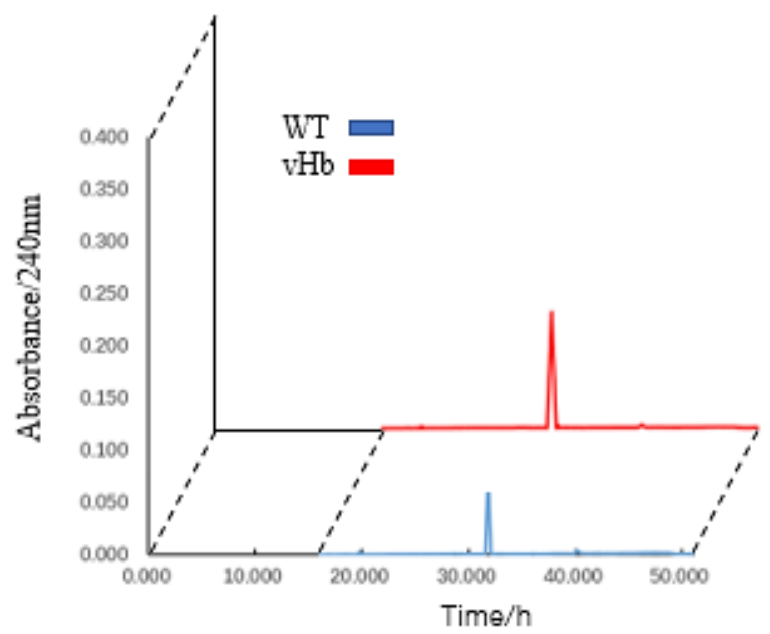

Fig. 5 HPLC absorbance profile of purified syringomycin. It shows the analysis of syringomycin yield of HS191- $\mathrm{vgb}$ and wild-type by HPLC, and it shows that the $V H b$ promotes the production nearly $71.05 \%$.
3, HS191-vgb grew faster than the wild-type, and its biomass was significantly elevated. It was worth noting that the mutant strain HS191-vgb reached stationary phase at around $14 \mathrm{~h}$, which was about 1 $\mathrm{h}$ later than the wild-type strain.

\section{Autoinduction of $\mathrm{VHb}$ improved the production of syringomycin}

To determine the effect of $v g b$ expression on syringomycin production, syringomycin bioassay was carried out to directly reflect the yield of syringomycin. As shown in Fig. 4, the G. candidum inhibition zone around HS191-vgb was enlarged from $2.5 \mathrm{~mm}$ to $3.2 \mathrm{~mm}$. Furthermore, the increased production of syringomycin was further confirmed using HPLC. According to the HPLC profile, Pseudomonas syringae HS191 secreted more syringomycin after the expression of $v g b$ gene (Fig. $5)$.

\section{Discussion}

Titer improvement is one of the key issues in metabolic engineering, the advances in synthetic and systems biology have allowed reprogramming of secondary metabolism. As an important source of secondary metabolites, $P$. syringae encodes a large variety of antibiotics [20,21], and $v g b$-mediated oxygen supply has been proven to be beneficial for fermentation [22-24]. The discoveries that the syringomycin synthetase $\mathrm{SyrB2}$ is an $\mathrm{O}_{2}$-dependent halogenase led us to speculate that the production of syringomycin could be improved by increasing the oxygen supply. The physiological investigation discovered that $P$. syringae propagated faster in the hypoxia condition during the logarithmic phase; however, the general cell yield was sacrificed. On the contrary, hyperoxia fermentation of $P$. syringae resulted in improved biomass during the stationary phase, but the propagation rate during the logarithmic phase was reduced [14]. From a practical point of view, a timely supply of oxygen during the stationary phase may facilitate the general cell yield and secondary metabolite production. The dynamic expression character of the quorum-sensing system serves as a switch for gene activation.

In this study, we established an autoinducible expression system for the controlled oxygen supply during fermentation, by combining the Vitreoscilla hemoglobin gene ( $v g b)$ and the LuxILuxR quorum-sensing system. The LuxI-LuxR quorum sensing system relies on the expression of the signaling molecule, Acyl-homoserine lactone 


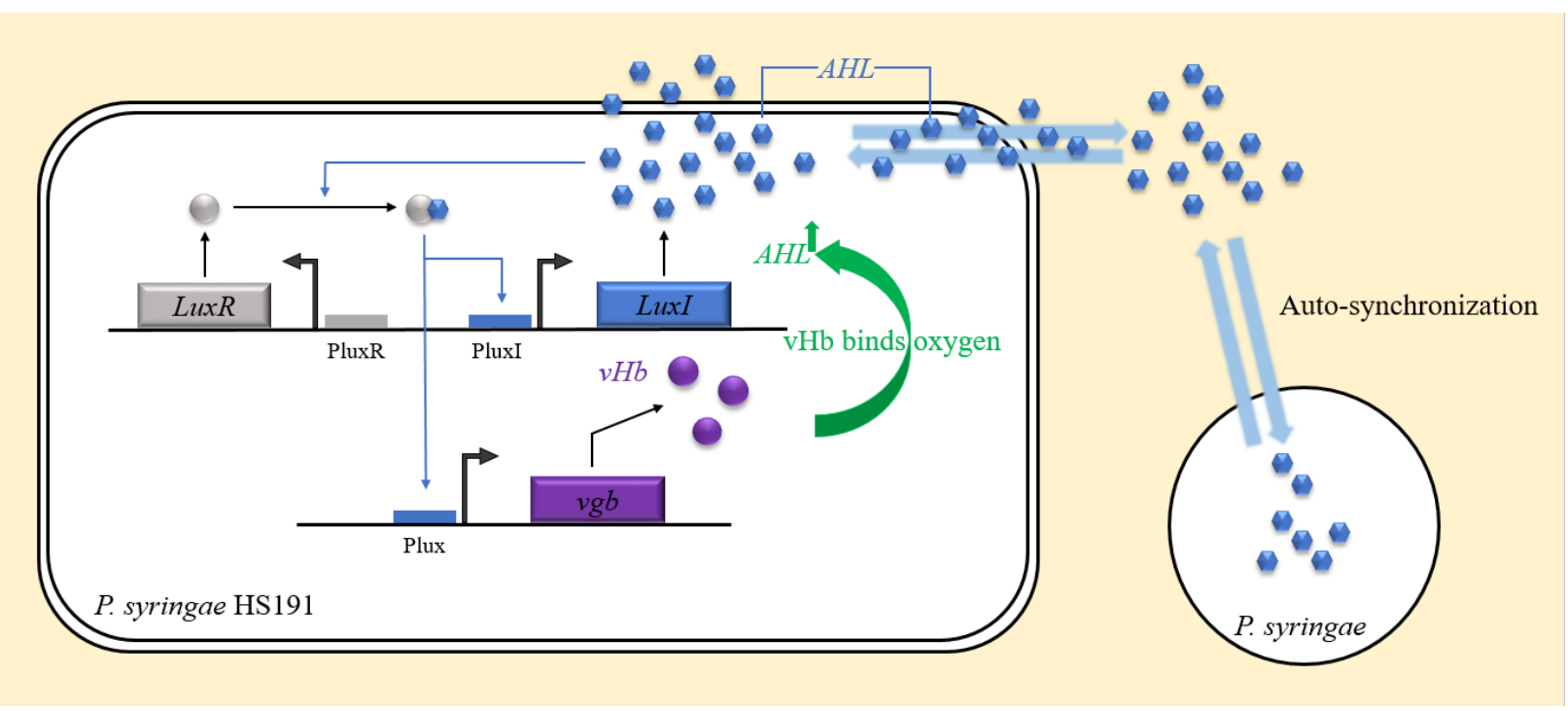

Fig. 6 Mechanism of $v g b$ gene in Pseudomonas syringae HS191. N-acyl-homoserine lactones (AHL) is a signal molecule in the incubation of HS191, which accumulates with the growth of the host strain and binds with LuxR to activate the Plux promoter when the bacteria enter the stationary phase of growth.

(AHL), which binds with the transcription regulator LuxR to function as an activator for the transcription of Plux promoter. The AHL was weakly expressed during the logarithmic growth and was not able to activate the $v g b$ gene until the stationary phase. The results showed that autoinduction of $\mathrm{VHb}$ not only increased the growth and biomass of strain HS191, but also improved the titer of syringomycin. The sfGFP reporter gene was integrated in-frame to the downstream of PsyrB1 promoter to monitor its expression profile. The fluorescence persistent period of the $v g b$-expressing strain HS191-GV was 27\% longer than that of strain HS191-sfGFP. The $v g b$-expressing strain HS191GV emitted green fluorescence about 30 min later than HS191-sfGFP, while the general fluorescencelasting time was $1 \mathrm{~h}$ longer than this counterpart.

Taking the growth dynamic into consideration, HS191-vgb grew faster than the wild-type, and its biomass was significantly elevated. It was worth noting that the mutant strain HS191-vgb reached stationary phase at around $14 \mathrm{~h}$, which was about 1 $\mathrm{h}$ later than the wild-type strain. According to the syringomycin bioassay data, the enlarged $G$. candidum inhibition zone around HS191-GV directly suggested the elevation in syringomycin secretion, which was then confirmed by HPLC analysis. In general, autoinduction of $\mathrm{VHb}$ resulted in a $71.1 \%$ increase in syringomycin production. These results demonstrated that the controlled oxygen supply by autoinduction of $\mathrm{VHb}$ opened a new avenue for the optimization of Pseudomonas fermentation and can probably be applied to other bacteria.

\section{Conflict of interest}

The authors declare no conflict of interest.

\section{References}

[1] Skerman VBD, McGowan V, Sneath PHA. Approved lists of bacterial names. Int J Syst Evol Microbiol 1980; 30(1):225-420.

[2] Stark BC, Dikshit KL, Pagilla KR. The biochemistry of Vitreoscilla hemoglobin. Comput Struct Biotechnol J 2012; 3(4):1-8.

[3] Kallio PT, Kim D, Tsai ps, Bailey JE. Intracellular expression of Vitreoscilla hemoglobin alters Escherichia coli energy metabolism under oxygenlimited conditions. Eur J Biochem 1994; 219 (1-2):201208.

[4] Lara AR, Jaén KE, Sigala JC, Mühlmann M, Regestein L, Büchs J. Characterization of endogenous and reduced promoters for oxygen-limited processes using Escherichia coli. ACS Synth Biol 2016; 6(2):344-356.

[5] Liu D, Wan N, Zhang F, Tang YJ, Wu SG. Enhancing fatty acid production in Escherichia coli by Vitreoscilla hemoglobin overexpression. Biotechnol Bioeng 2017; 114(2):463-467.

[6] Khosla C, Bailey JE. The Vitreoscilla hemoglobin gene: molecular cloning, nucleotide sequence and genetic expression in Escherichia coli. Mol Gen Genet 1988; 214(1):158-161.

[7] Luo Y, Kou X, Ding X, Hu S, Tang Y, Li W, Xia L. Promotion of spinosad biosynthesis by chromosomal integration of the Vitreoscilla hemoglobin gene in Saccharopolyspora spinosa. Sci China Life Sci 2012; 
55(2): 172-180.

[8] Li W, Rokni-Zadeh H, De Vleeschouwer M, Ghequire MG, Sinnaeve D, Xie GL, De Mot R. The antimicrobial compound xantholysin defines a new group of Pseudomonas cyclic lipopeptides. PloS one 2013; 8(5):e62946.

[9] Roongsawang N, Washio K, Morikawa M. Diversity of nonribosomal peptide synthetases involved in the biosynthesis of lipopeptide biosurfactants. Int $\mathrm{J}$ Mol Sci 2010, 12(1), 141-172.

[10] Shi T, Xie J. Regulation mechanisms underlying the biosynthesis of daptomycin and related lipopeptides. J Cell Biochem 2012; 113(3):735-741.

[11] Dayan FE, Duke SO. Natural compounds as nextgeneration herbicides. Plant Physiol 2014; 166(3):1090-1105.

[12] Das P, Mukherjee S. Sen R. Genetic regulations of the biosynthesis of microbial surfactants: an overview. Biotechnol Genet Eng Rev 2008; 25(1):165-186.

[13] Vaillancourt FH, Yin J, Walsh CT. SyrB2 in syringomycin $\mathrm{E}$ biosynthesis is a nonheme FeII $\alpha$ ketoglutarate-and $\mathrm{O}_{2}$-dependent halogenase. Proc Natl Acad Sci USA 2005; 102(29):10111-10116.

[14] Clark DS, Burki T. Oxygen requirements of strains of Pseudomonas and Achromobacter. Canadian J Microbiol 1972; 18(3):321-326.

[15] Vidaver AM. Synthetic and complex media for the rapid detection of fluorescence of phytopathogenic pseudomonads: effect of the carbon source; 1967.

[16] Sambrook J, Fritsch EF, Maniatis T. Molecular cloning: a laboratory manual (No. Ed. 2). Cold spring harbor laboratory press; 1989.

[17] Swingle B, Bao Z, Marke E, Chambers A, Cartinhour S. Recombineering using RecTE from Pseudomonas syringae. Appl Environ Microbiol 2010; 76(15):49604968.

[18] Bidwai AP, Zhang L, Bachmann RC, Takemoto JY. Mechanism of action of Pseudomonas syringae phytotoxin, syringomycin stimulation of red beet plasma membrane ATPase activity. Plant Physiol 1987; 83(1):39-43.

[19] Raaijmakers JM, de Bruijn I, de Kock MJ. Cyclic lipopeptide production by plant-associated Pseudomonas spp.: diversity, activity, biosynthesis, and regulation. Mol Plant-Microbe Interact 2006; 19(7):699-710.

[20] Yu H, Shi Y, Zhang Y, Yang S, Shen Z. Effect of Vitreoscilla hemoglobin biosynthesis in Escherichia coli on production of poly ( $\beta$-hydroxybutyrate) and fermentative parameters. FEMS Microbiol Lett 2002; 214(2):223-227.

[21] Raaijmakers JM, Weller DM, Thomashow LS. Frequency of antibiotic-producing Pseudomonas spp. in natural environments. Appl Environ Microbiol 1997; 63(3):881-887.

[22] Chen YM, Xu HY, Wang Y, Zhang JF, Wang SM. Vitreoscilla hemoglobin promotes Salecan production by Agrobacterium sp. ZX09. J Zhejiang Uni Sci B 2014: 15(11):979-985.

[23] Ma R, Lin X. Vitreoscilla hemoglobin gene (vgb) improves lutein production in Chlorella vulgaris. Chinese J Oceanol Limnol 2014; 32(2):390-396.

[24] Liang F, Shouwen C, Ming S, Ziniu Y. Expression of Vitreoscilla hemoglobin in Bacillus thuringiensis improve the cell density and insecticidal crystal proteins yield. Appl microbiol Biotechnol 2007; 74(2):390-397. 